\title{
"ENTRE LAS COSAS COTIDIANAS": EL APARECER DEL MUNDO SENSIBLE DESDE LA FENOMENOLOGIA TEMPRANA ${ }^{1}$
}

\author{
Felipe Johnson ${ }^{2}$
}

\begin{abstract}
Resumen: Este artículo se propone describir el modo de aparecimiento del mundo sensible, entendiéndolo como el primer suelo sobre el cual la vida humana se instala efectivamente entre cosas concretas. Se intentará desvincular este nivel de presencia inmediata y cotidiana del mundo exterior del modo como tradicionalmente se le concibe, a saber, en el sentido de una manifestación "objetual". En este contexto, se recurrirá a descripciones fenomenológicas de la percepción que tuvieron lugar principalmente en trabajos filosóficos y psicológicos de la primera mitad del siglo XX, y que fueron influenciados por las reflexiones tempranas de Edmund Husserl. A partir de estas obras se indagará en la manera como el mundo sensible comparece a la vida cotidiana, al margen de todo carácter de "verdad epistémica". Así, nuestro propósito será comprender el mundo exterior en una íntima relación con una situación vital, en la cual la vida abre su propio suelo para poder vivir.
\end{abstract}

Palabras clave: Percepción. Cualidad sensible. Mundo exterior. Cotidianidad. Fenomenología temprana.

\section{INTRODUCCIÓN: SOBRE LAS PRETENSIONES DEL PRESENTE ESTUDIO}

Estas reflexiones se proponen indagar en el modo como el objeto sensible se presenta en el acto de la sensibilidad humana. Para tales fines quisiéramos retomar una discusión que tuvo lugar en la psicología a principios del siglo XX, y que resultó ser una respuesta a la tradición fisiológica dominante en ella, animada por la expansión del positivismo en las ciencias en general. En este contexto, aparecen filósofos y psicólogos que intentan prescindir o,

1 http://dx.doi.org/10.1590/S0101-31732018000100008 Artículo elaborado en el marco del proyecto Fondecyt Regular n ${ }^{\circ}$. 1150034, del cual el autor es Investigador Responsable.

2 Profesor Jornada Completa del Área de Investigación Filosófica del Departamento de Ciencias Sociales, Universidad de La Frontera, Temuco, Chile. E-mail: felipe.johnson@ufrontera.cl.

Entre sus publicaciones cuentan: el libro Der Weg zum Leib: Ontologie der Leiblichkeit anhand des Denkens Martin Heideggers (2010) y artículos como "El cuerpo como posibilidad de la vida: el modo de despliegue del mundo concreto" (2011), "Corporalidad, sensibilidad y mundo: pensando la concreción del existir desde el pensamiento de Heidegger" (2014), "La problemática dimensión orgánica del cuerpo humano: sobre los límites entre la Analítica del Dasein de Martin Heidegger y el análisis del Dasein de Medard Boss" (2017), entre otros. 
al menos, restar protagonismo a interpretaciones netamente orgánicas, para llevar a cabo una descripción acabada de los estados puros e inmanentes de la conciencia, concibiéndola como una psicología fenomenológica. Así, las presentes discusiones referirán a trabajos directa o indirectamente influidos por Edmund Husserl, ya sea mediante la primera edición de Investigaciones lógicas (1901), como por lecciones dictadas por él en el marco de dicha obra. Es en dicha edición donde Husserl se muestra próximo a la idea de Brentano de una psicología descriptiva y que venía siendo el sentido de su investigación desde su tesis de habilitación: Sobre el concepto de número (1887). Esta fase temprana de la investigación husserliana tendrá como horizonte la descripción de la vivencia inmanente, en el intento de indagar en las condiciones subjetivas de la aparición de los objetos, como paso anterior a una explicación causal en el sentido de una psicología genética. En la introducción a esta primera edición Husserl manifestará que la "Fenomenología es psicología descriptiva." Y a continuación agregará: "Entonces bien, la crítica del conocimiento ha de ser esencialmente psicología o al menos construida sólo sobre el suelo de la psicología." (HUSSERL, 1992a, p. 24). Suprimiendo afirmaciones en esta dirección, es la segunda edición, en efecto, aquella que reflejará el programa husserliano de una fenomenología, en la cual el análisis psicológico se reformulará en un análisis trascendental, aprehensor de las esencias de la conciencia (PEUCKER, 2002, p. 160-188).

En efecto, los análisis tempranos de Husserl acerca de la percepción trazan una línea de investigación que parecía rendir cuenta de su carácter activo en la constitución del objeto percibido, en oposición a la idea de percepción en cuanto una recepción pasiva de meros datos. La comprensión de la percepción como "acto objetivante" (objektivierender Akt) cuyo carácter fundamental es el de donar su propio objeto en sí mismo, denominado por Husserl (1992a, §38) "acto ponente" (setzender Akt), ya era una tesis fundamental desarrollada en esta primera edición de Investigaciones lógicas. Así, Husserl abordará el problema de la percepción sensible en vistas a tres momentos constitutivos, como son la cualidad, es decir, el modo como percibimos (afirmativa, dubitativa o refutativamente), la materia o "sentido de aprehensión" (Auffassungssinn), i.e., el énfasis en que el objeto es percibido (el árbol como ser vivo o como fuente de sombra), y su contenido representativo (repräsentierender Inhalt), en este caso, del objeto mismo (HUSSERL, 1992b, p. 620).

En este contexto, el problema de la "sensación" (Empfindung) se vuelve una tarea fundamental de análisis. Y es que estas investigaciones dan lugar a 
comprender que las cualidades sensibles no constituyen un cúmulo de datos reunidos, cuya suma daría lugar al objeto, "[...] como si las cosas que aparecen en palabras de Husserl (1992b, p. 764) - fueran una complexión de contenidos de sensaciones." Ante todo, la donación del objeto sensible obedece a un acto que deja ver al objeto mismo en su propia plenificación sensible. En este sentido, una reformulación de las sensaciones implicará entenderlas en su rol presentativo del objeto percibido, y no como datos anexos a él, es decir, como afecciones puramente subjetivas, que se harían presentes junto a la percepción. Así, la tesis central de Husserl será que las sensaciones mismas no son percibidas. "No veo sensaciones de colores, sino cosas coloridas, no oigo sensaciones de sonidos, sino la canción de la cantante, etc." (HUSSERL, 1992a, p. 387), advierte éste para destacar que la cualidad sensible tiene una función específica como es la de ser aquellos contenidos presentativos (präsentierende Inhalte) o sensaciones presentativas (präsentierende Empfindungen) (HUSSERL, 1992a, p. 397), que en un objeto percibido determinado son "aprehendidos" como sus propiedades objetuales (HUSSERL, 1992b, p. 766). De esta manera es como en la dirección de una psicología descriptiva viva en la primera edición de Investigaciones lógicas los autores a estudiar entenderán que el problema de la sensación ha de ser tratado en su función expositiva, al modo, por cierto, de una descripción inmanente del fenómeno de la percepción, es decir, a la base de una reflexión de las vivencias psíquicas.

En efecto, es sabido que en la ciudad de Göttingen se constituyó alrededor de Husserl un círculo fenomenológico concentrado principalmente en el problema de la percepción y de la constitución del objeto en ella (PLESSNER, 1985, p. 348). Motivados por estas problemática de la constitución del objeto en el acto de la percepción, emergen trabajos dirigidos por el mismo Husserl, como Aportes para una fenomenología de la percepción de Wilhelm Schapp, o Investigaciones sobre el concepto de sensación de Heinrich Hofmann, los cuales se centrarán en el problema de las cualidades sensibles como presentadoras del objeto percibido. ${ }^{3}$ En el ámbito de la psicología, David Katz desarrollará estudios sobre la donación táctil, recurriendo a una investigación inmanente del acto de tocar. De manera indirecta, aunque

3 Hofmann (1913, p. 85) mismo señalará que el concepto de "constitución" en sus propias investigaciones perceptivas correspondería al modo como Husserl lo habría usado, dice él: "[...] reiteradamente en sus lecciones y en sus discusiones privadas." Por su parte, Schapp (2013, p. 16) dirá: "Queremos, por lo pronto, hacer valer algo en tanto que percibido, cuando se nos muestra sensiblemente, cuando está 'en cuerpo presente' (leibhaftig) ante nosotros, como Husserl procuraba decir en sus lecciones." 
suscribiendo tesis en la dirección de una psicología descriptiva, aparecen también estudios de las sensaciones olfativas, como en El olor (1916) de Hans Henning. Decisivos resultan estos trabajos, junto a otros que serán contemplados a lo largo de este estudio, ya que proporcionarán referencias a casos perceptivos que otorgan a las presentes discusiones sobre el mundo sensible un suelo fenoménico concreto en el cual apoyarse. En este contexto, sus análisis serán el centro de las siguientes discusiones, y pese a que las tesis fundamentales de estos autores se encuentran ligadas a las tesis de la percepción de Husserl, su propio pensar deberá quedar implícito de modo que las consideraciones de este grupo de pensadores, en parte psicológicas, en parte filosóficas, adquieran protagonismo. Cuando sea oportuno, sin embargo, se indicarán con más precisión sus coincidencias temáticas. ${ }^{4}$

Por esta misma vía, cabe señalar, además, que a continuación no se pretenderá realizar una revisión histórica de este particular estudio de la psyché. Más bien, estos trabajos serán tomados como una fructífera fuente de descripciones muy precisas, que, elaboradas en una constante referencia a casos perceptivos particulares, pueden contribuir a un esbozo inicial de una comprensión situacional de la aparición sensible del mundo. En efecto, atender a estas descripciones, a nuestro juicio, se traduce, en última instancia, en disponer del suelo concreto de la aparición sensible para comprender cómo es que la vida humana misma que es y habita en una situación comprensiva de su mundo, abre las cosas con las que ella trata y así configura su aparición más cotidiana. Tal tesis pareciera ser el paso siguiente de las consideraciones fenomenológico-psicológicas que acá serán expuestas, no obstante, aunque los autores tratados no lo dieron, sí es posible apreciar cómo desde el ámbito de la concretud descrita parece anunciarse tal carácter situacional. Nuestro interés será, en definitiva, tomar dichos estudios para discutir sobre la percepción, aclarar las razones de este tratamiento particular en el contexto de su oposición a la perspectiva fisiológica dominante, ganar claridades acerca de la tendencia de sus propias tematizaciones y examinar cómo es posible realzar una perspectiva situacional de la manifestación del mundo sensible. Con esto, nuestro interés último será acercarnos al suelo más concreto de nuestra vida, aquel de las cosas sensibles, entre las cuales, ciertamente, a la vida humana le es dado desplegarse en su inmediatez.

\footnotetext{
${ }^{4}$ Para un análisis detallado de las principales tesis acerca de la percepción en Husserl, remitimos a la V investigación lógica: "Über intentionale Erlebnisse und ihre 'Inhalte”" (1992a) y a su lección de 1907, Ding und Raum (1973). Asimismo, Mulligan (1995) realiza una exposición detallada de los aspectos centrales de la percepción en Husserl en el programa temprano de su psicología descriptiva.
} 


\section{LA NOCIÓN DE SUBSTANCIA A LA BASE DE LA CONCEPCIÓN FISIOLÓGICA DE LA PERCEPCIÓN}

Por cierto, aún en la actualidad es posible advertir las tesis dominantes de una concepción fisiológica de la percepción. Y es que todavía se considera que el objeto del mundo exterior sería visto cuando emanan destellos de luz, entendidos como frecuencias de ondas lumínicas que se proyectarían en nuestros ojos. Enfocados éstos en la retina, excitarían millones de bastones y conos, receptores que, por su parte, transformarán la energía luminosa en un tipo de energía eléctrica húmeda. Conducida por el nervio óptico hasta el cerebro, tal energía constituiría una determinada red neuronal, la cual sería reconocida por el cerebro como el objeto observado, mediante un así llamado "procesamiento neuronal" (GOLDSTEIN, 2005, p. 5).

Tal explicación, por cierto, no es del todo nueva. La tesis fundamental se encuentra ya en Las pasiones del alma (1649), obra en la cual Descartes (1997, p. 91 s.) distingue que:

Las [percepciones] que referimos a las cosas que existen fuera de nosotros, a saber, a los objetos de nuestros sentidos, son causadas [...] por esos objetos, los cuales, provocando movimientos en los órganos de los sentidos exteriores, los provocan también en el cerebro a través de los nervios que hacen que el alma los sienta.

Tanto en la actualidad como para Descartes, el mecanismo de la percepción resulta ser sumamente complejo. Pero no menos complejo es el mundo que, en última instancia, parece excitarlo. Ondas de luz, en el caso de la vista, frecuencias de sonido, en la audición, reacciones químicas, para el gusto y el olor, describen un entorno que se encuentra bombardeando constantemente una cantidad ingente de señales físicas que chocan con organismos dispuestos a recibirlas. Al menos, esa es la realidad que Descartes, en su momento, y, posteriormente, una parte importante de la psicología, han logrado descifrar. ${ }^{5}$ Desde sus consideraciones, el modo como la vida humana entra en un comercio con tal realidad se concibe de una manera muy

\footnotetext{
${ }^{5}$ Las influencias del pensar cartesiano en la psicología son destacadas con precisión por Erwin Straus en su obra Vom Sinn der Sinne. En en ella se subraya el influjo de Descartes en la subestimación de las sensaciones tomadas en su rol cognitivo (STRAUSS, 1978, p. 9 s.), en la noción inmanentista de las sensaciones (STRAUSS, 1978, p. 213-216), en la concepción mecánica, fisiológica, de los procesos psicológicos (STRAUSS, 1978, p. 275-279) y en la definitiva deshumanización de la Naturaleza, y con ello, de los fenómenos humanos mismos, en el proyecto de establecer los fundamentos de su física (STRAUSS, 1978, p. 195 s.).
} 
particular: la percepción pareciera acaecer en cuanto este mundo afecta, en un sentido eminentemente causal, al sujeto perceptivo.

Pero, ante todo, ¿cómo pudo llegar Descartes a entender la realidad como causa de nuestras percepciones? Cuando observamos una piedra o escuchamos una melodía, atendemos a colores, sonidos, mas entre tales propiedades no hallamos alguna que pueda ser llamada "causa". En estos casos, la percepción no parece ser responsable de tal expresión. Y es que el postulado de la causalidad es de una naturaleza muy diferente a sostener, por ejemplo, que la piedra vista sea de color gris. Mientras que otras piedras pueden presentar otros colores, la causalidad es una proposición válida para cada relación perceptiva particular. La causalidad es una ley universal, y dichas leyes, Descartes lo sabe bien, no se obtienen mediante los sentidos, sino mediante la razón. Así, en Meditaciones metafísicas, la primera noción común o axioma que fundará los resultados ahí obtenidos, y que Descartes expondrá según el modo geométrico, atendiendo a las demandas realizadas por filósofos y teólogos a su obra, reza: “[...] no hay ninguna cosa existente de la cual no se pueda interrogar cuál es la causa por la que ella existe." (DESCARTES, 1996b, p. 127).

Según el modo como la geometría procede, entonces, la relación causal de las partes, como es el caso de la aparición de sensaciones en nosotros, puede ser deducida del axioma que establece que no hay nada sin una causa anterior que le haya dado su existencia. La formulación positiva diría, por tanto, que ha de darse, primero, la causalidad, como legalidad total de la realidad, para entender, luego, que sus partes, las cosas del mundo, han de comportarse entre ellas causalmente. Dicho supuesto no es menor para una comprensión de la percepción. Y es que éste expresa que hay una incidencia de una instancia de la relación en la otra, en nuestro caso, la luz emanada por el objeto exterior produce el movimiento de los nervios de quien percibe.

Empero, la causalidad no sólo supondría la incidencia del objeto en el sujeto. A su vez, ésta parece contener una caracterización de las cosas mismas comprometidas en tal relación, al establecer justamente una separación entre las instancias en juego: la causa, por un lado, y, por otro, el efecto. Se requiere, a saber, que éstas no sólo estén separadas, sino que, a su vez, existan autónomas respecto de la otra. Y es que sería inviable pensar que ambas instancias son una sola, ya que, en palabras de Tomás de Aquino (2001, p. 112), resulta imposible "[...] que algo sea causa eficiente de sí mismo, pues sería anterior a sí mismo." Se obtiene, entonces, que el objeto exterior, la causa del movimiento, existe, y con independencia del sujeto que percibe. Así, ambas instancias de la relación, 
en palabras de Descartes (1996a, p. 47), “[...] no requieren más que de sí mismas para existir." En suma, ambas son substancias.

Entonces bien, si la causalidad es una caracterización de la realidad en general, su requisito, la substancialidad, ha de ser igualmente universal. En los términos que nos interesan, diremos, entonces, que las cosas del mundo, en tanto que substancias, poseerían una estructura que en si misma contiene su propio ser. Las cosas del mundo se entienden, por tanto, en un retraimiento hacia sí. Esto confirmaría, ciertamente, que su auténtica realidad ha de hallarse necesariamente en esta estructura interna que sustentaría su existencia. Mas, al igual como ocurre con la causalidad, mediante los sentidos tampoco conocemos tal substancia. Nuestra relación con ésta viene a ser mediada más bien por sus cualidades, es decir, por las maneras según las cuales se aparece. Se trata del color, el sabor, esto es, "efectos" dados en el sujeto, que le indicarían que el objeto está ahí fuera, existiendo (DESCARTES, 1996a, p. 47), pues aquellos movimientos de los nervios, y que es lo que finalmente da lugar a la percepción de las diversas cosas, depende necesariamente de la causa que los ha provocado. Así, todas las cosas de la realidad se compondrían de un substrato en el cual habita su auténtica realidad, y en tanto que substancias, todas poseerán cualidades dependientes de la presencia de dicho fundamento (DESCARTES, 1996a, p. 49).

Así es como las afirmaciones de Descartes se vuelven ejemplares para comprender la idea de percepción como un acto necesariamente pasivo por parte del sujeto cognoscente. Tal necesidad se explica desde una concepción total de la realidad como substancia, i.e., entendiendo que los entes particulares, retraídos en sí mismos respecto de su ser, afectan causalmente a otros entes. En este contexto, no hay razón alguna para suponer que haya en el sujeto cognoscente algún elemento activo que intervenga en la configuración de lo percibido. Es el objeto el que ha de proporcionar sus propios datos. El sujeto perceptivo no es más que un "receptor" en cuya alma puede o no configurarse una imagen fiel de lo que recibe. Así, pues, la actual concepción de la percepción como recepción pasiva de estímulos externos parece fundarse en una comprensión total de la realidad, en la cual, como se advierte desde Descartes, primaría la noción de substancia como su guía.

Asimismo, desde la destacada pasividad de la percepción es posible advertir que el acto de observar las cosas del mundo exterior ha de ser sumamente cauteloso. Como precisa Descartes, cuando percibimos, debemos distinguir en lo percibido aquellos caracteres que pertenecen a la substancia, en el caso 
del mundo exterior, a la res extensa, esto es, al largo, ancho y profundidad, de las sensaciones, como el color o sabor (DESCARTES, 1996a, p. 54). Y es que las sensaciones son cambiantes y pueden conducir a una aprehensión confusa de lo visto, mientras que son las primeras aquellas que refieren la naturaleza de lo que observamos (DESCARTES, 1996a, p. 65). No se trata, pues, para el sujeto, de atender al rojo del objeto, sino, ante todo, de entender que tal color no es posible sin una extensión que lo sustente. Sólo entonces se advertirá su auténtica realidad.

Así, si las cosas de la realidad no fuesen substancia, bastaría tan sólo con lo que vemos u oímos. No obstante, como su realidad auténtica se retrae a su estructura interna, ha de exigírsele a la percepción que, entre la gran cantidad de datos que nos proporciona, alguno sea verdadero, o sea, que se corresponda con esa estructura propia del objeto conocido. Antes de que el sujeto emita juicios acerca de lo que observa en el exterior, debe acaecer, de modo necesario, una adaequatio intellectus ad rem. La realidad de la substancia, entonces, impone a la percepción un valor de verdad que debe satisfacer o, lo que es lo mismo, la noción de percepción se subsume a las exigencias propias del acto del conocimiento, i.e., al requisito de la veracidad. Con todo, el fenómeno de la sensibilidad se vincula necesariamente con el problema del acceso a la auténtica realidad, aquella que estaría contenida en la estructura autónoma de las cosas exteriores.

Así es como estas discusiones acerca de la substancia en Descartes advierten que el sujeto percipiente se traduce, en último término, en un sujeto cognitivo. La percepción es interpretada como si quien observase, tocase u oyese, lo hiciese para obtener notas efectivas de su entorno, para, así, poder emitir juicios con valor de verdad. Justamente, tal exigencia epistémica y el carácter pasivo desde los cuales hoy se plantea la sensibilidad son los que parecen emerger al interior de la concepción substancial del mundo. Por tanto, atendiendo a lo anterior es que la tarea que nos proponemos realizar dice relación con indagar en tales supuestos, para examinar, por lo pronto, si es que ellos contribuyen a una caracterización del auténtico aparecer del mundo sensible.

\section{LA PERCEPCIÓN EN EL HORIZONTE DE LA VIDA CORRIENTE}

Decíamos, pues, que nociones tales como "causalidad" y "substancia" daban lugar a un modo concreto de concebir la percepción. 
Mas, también se discutía que ellas mismas no son percibidas, i.e., no pertenecen al ámbito de lo sensible, como, en efecto, podemos considerar cualidades como colores, aromas, etc. En este sentido, se advierte que tales nociones, aunque decisivas para una caracterización de la percepción, no son aspectos obtenidos a partir de un examen concreto de alguna experiencia perceptiva particular, por lo cual, difícilmente podríamos sostener que "causalidad" o "substancia" corresponden a intentos descriptivos de este acto tan propio del sujeto. Y es que, más bien, se trata de supuestos explicativos de cuyo auténtico derecho nada se puede decidir sino hasta observar el propio mundo de la percepción. Dicha advertencia nos prevendría, así, de admitir sin más tales nociones en tanto que externas al fenómeno que nos ocupa.

Ahora bien, estos supuestos conceptuales no son únicamente los que se encuentran en juego en una caracterización de la percepción. Otros aspectos inciden igualmente en el modo como se le concibe y los hallamos, de hecho, en las explicaciones del mismo Descartes, como en gran parte de la tradición fisiológica de la psicología, a saber, que los fenómenos psíquicos, como lo es el mismo acto de percibir, son la expresión de determinadas configuraciones nerviosas. ${ }^{6}$ Precisamente, este es el problema que fue considerado en los estudios psicológicos a principios del siglo XX. En dicha época, como seńalábamos, emerge una tendencia en la psicología que aboga por un estudio íntimo de los fenómenos psíquicos. Se trata de una reformulación de la Psicología de los sentidos, al margen de un estudio del sistema nervioso, salvaguardando una descripción inmanente de los contenidos de conciencia, ${ }^{7}$ comprendiendo que la percepción sensible o exterior, al modo como Husserl lo destaca en Investigaciones lógicas (1901), puede ser entendida también en la evidencia de la inmanencia (HUSSERL, 1992b, p. 769).

\footnotetext{
${ }^{6} \mathrm{Al}$ respecto, es oportuno atender a las críticas que hará Erwin Straus a la concepción fisiológica dominante en la psicología. Éste devela los supuestos positivistas que subyacen a las interpretaciones de la conducta humana. Ciertamente, el principal objetivo de las críticas se dirige a la teoría conductista de Pawlow (STRAUS, 1978, p. 28-80), sin embargo, avanzada la exposición, se advierte cómo la Psicología de la Gestalt también insistiría en suscribir la tesis de una correspondencia entre vivencias y procesos fisiológicos, en su epifenomenalismo. Straus destaca, así, que dicha teoría no termina por desvincularse del cartesianismo ya instalado en esta disciplina (STRAUS, 1978, p. 317-322).

7 El concepto de conciencia entendida como totalidad unitaria de las vivencias intencionales en tanto que "[...] fajo o entretejido de las vivencias psíquicas" (HUSSERL, 1992a, p. 356) destacado en la primera edición de Investigaciones lógicas, y reescrito en la segunda, es justamente el punto de partida para estas consideraciones de carácter psicológico fenomenológico y, por ende, se tratará de análisis centrados en el sujeto empírico (MARBACH, 1974, cap. I).
} 
En 1908, August Messer, entendiendo que la percepción es un acto donador de objeto, ${ }^{8}$ hará énfasis en la eminente distancia entre los análisis estrictamente psicológicos y los propios de las ciencias naturales: "No vemos éter o nuestra retina, sino que vemos justamente 'verde'; y en esta sensación no hay nada así como procesos químicos o eléctricos en nervio y cerebro." (MESSER, 1908, p. 12). Y es que el estudio fisiológico tiende a descomponer la percepción sensible en un cúmulo de sensaciones que luego puedan ser reconducidas a diversos receptores nerviosos, al modo de lo que podría denominarse, como lo hace David Katz en su obra La construcción del mundo táctil (1925), una "atomización" del ámbito de la sensibilidad, en un sentido "histiológico" (KATZ, 1925, p. 58). ${ }^{9} \mathrm{Al}$ igual que a Messer, la preocupación por garantizar la escisión entre ciencia natural y psicológica, lleva a Katz (1925, p. 11) a advertir que "[...] la mayoría de los hombres puede morir, sin que alguna vez hayan vivenciado la estimulación de un punto de presión o de calor o un auténtico umbral sensible."

El mundo de la percepción exige más bien ser entendido desde aquello que él mismo ofrece, i.e., las cualidades propias vividas en él. En el verde, en la aspereza, no hallamos nada así como hechos o procesos físicos. Toda reconducción a ellos termina por arrojar la reflexión a una esfera ajena al

\footnotetext{
${ }^{8}$ Messer entenderá el problema de la percepción sensible y las sensaciones en términos de una vivencia intencional en tanto instancia subjetiva constituyente del objeto percibido. En constante referencia a Investigaciones lógicas, sus análisis concebirán a la sensación (Empfindung) como representante (repräsentierend) donado en virtud de la materia del acto perceptivo, esto es, su sentido de aprehensión (Auffassungssinn) y, por ende, fundada en una "intención animadora" (beseelende Intention) (MESSER, 1908, p. 57-63). Este punto de partida le permitirá advertir que los análisis inmanentes de las vivencias perceptivas han de tener por objeto comprender aquella relación entre sensación (Empfindung) y pensar (Denken), término mediante el cual se seńala a un tipo fundamental de acto objetivante (objektivierender Akt), como es la percepción sensible, (MESSER, 1908, p. 69) cuyas reglas sería necesario entender mediante un estudio de la experiencia inmanente.

9 La oposición a las explicaciones fisiológicas es una posición característica de estas descripciones inmanentes de la percepción. Como Katz, Heinrich Hofmann discute también si la "psicología de los sentidos" (HOFMANN, 1913, p. 2) pertenecería a la psicología o a la fisiología. Para él, tal problema hallaría una solución si se advierte que el objeto de la psicología no se confunde con el de las ciencias naturales. Esto le lleva a defender una descripción "[...] puramente psicológica” (HOFMANN, 1913, p. 12), adoptada de la obra de Messer. Destaca, en este contexto, el reconocimiento que estos autores hacen al psicólogo Ewald Hering. En sus discusiones sobre la dependencia entre las sensaciones y determinados procesos fisiológicos, éste distingue la "sensación visual" (Gesichtsempfindung) de una "substancia visual" (Sehsubstanz) (HERING, 1878, p. 72), i. e., dos dimensiones aparentemente vinculadas, lo psíquico y lo físico, pero de cuya unión efectiva no habría claridad. Tales son las críticas de Hering a la psicofísica de Fechner (HERING, 1878, p. 73), desde la cual parecen gestarse estos esfuerzos en la psicología por escindir el mundo dado en la conciencia de aquel entendido como un mero objeto exterior. Interesante resulta esta distinción entre el mundo de las ciencias naturales y el psicológico (HUSSERL, 1973, p. 3-7).
} 
fenómeno mismo de la sensibilidad, y, por ende, la investigación es arrebatada de su auténtico campo de estudio: aquel mundo sensible en cuanto vivido sensiblemente. Dicho de otra manera, la reacción frente a lo fisiológico, impotente, por su parte, de rendir cuenta de la experiencia de la sensibilidad en tanto que tal, acentúa la necesidad metódica de permanecer en la esfera de la conciencia, empero, de una manera particular. La conciencia a la que se apela es la que tiene lugar en el mundo mismo, cuando la vida humana se halla efectivamente entre las cosas, en su absoluta cotidianeidad: es una conciencia de la vida corriente. Es en ella donde el mundo se presentaría vitalmente. Así es como, en el contexto del llamado a escindir a la psicología de la fisiología, Heinrich Hofmann puede demarcar, en su trabajo Sobre el concepto de sensación (1912), el ámbito de estudio de la percepción visual atendiendo a la cosa visual misma (Sehding), i. e., a las "cosas, como ellas se nos aparecen en cada momento en el ver" (HOFMANN, 1913, p. 57), distinguiéndolas de la cosa real, o "cosa-átomo" (HOFMANN, 1913, p. 52), en el sentido "objetual" de las ciencias naturales. ${ }^{10}$

De esta manera se puede entender la desvinculación que ha de acaecer entre la explicación fisiológica y una consideración atenta de la sensibilidad. Mas, esto no tan sólo en razón de la lealtad a la cualidad misma del mundo sensible, sino, también, porque aquella aleja a la reflexión del ámbito donde la percepción auténtica acaece: la vida corriente. Ahora, ¿̨cuál es el beneficio real de acudir a la vida cotidiana en el estudio de la sensibilidad? Hofmann (1913, p. 53) lo expresa negativamente: "La cosa de la vida corriente no es, por cierto, una mera suma de propiedades particulares sensibles [...].” ${ }^{11}$ Así, este posicionamiento en la vida corriente vendría a garantizar, en última instancia,

\footnotetext{
${ }^{10}$ No quisiéramos dejar de mencionar la reseña de esta obra que realizara Ortega y Gasset para la Revista de Libros, en 1913. El artículo difunde dicha investigación en psicología, precisando cómo es que Hofmann abordaría el problema de las sensaciones fenomenológicamente. La relevancia de esta reseńa, no obstante, es que es el testimonio de la introducción de la palabra "vivencia" en la lengua espańola, como traducción del vocablo alemán "Erlebnis". Ortega agrega una nota al pie "pidiendo auxilio" (ORTEGA Y GASSET, 1959, p. 91 s.) a quienes tengan interés por la filosofía y a los filólogos, pues, en Alemania habría nacido un concepto para el cual no existiría un equivalente adecuado español. Éste confiesa no tener otra opción que buscar una nueva palabra, construyendo a partir de "vivir" el neologismo "vivencia", al modo como se construye "existencia" de "existir". Empero, a pesar de que se advierta que, por su novedad, tal palabra pueda parecer "mal sonante", hoy en día ésta resulta ser completamente natural y es parte no sólo del vocabulario de la fenomenología en habla hispana, sino de muchas áreas científicas en donde se discute sobre lo humano.

${ }^{11}$ Husserl (1992b, p. 767). dirá: "Cuando un objeto exterior es percibido (la casa), entonces en esta percepción las sensaciones presentativas [präsentierende Empfindungen] son vivenciadas, pero no percibidas."
} 
la obtención de un concepto positivo de la cosa misma, resguardándola, a cada momento, en su manifestación inmediata y cotidiana. Un estudio eminentemente psicológico de los sentidos permite elucidar, entonces, el mundo mismo de quien percibe. En esta dirección ha de entenderse, de hecho, la advertencia de Katz (1925, p. 55), al concebir su estudio de las percepciones táctiles en beneficio de una "teoría del objeto" (Gegenstandstheorie). El problema es, en definitiva, cómo es dicho mundo sensible; cuál es la naturaleza de las cosas que se manifiestan para quien sensiblemente habita entre ellas. En nuestros términos, se trata, por tanto, de entender cómo es que se aparece auténticamente aquel mundo inmediato para la vida humana concreta. Así es como la investigación de la sensibilidad deberá aclarase acerca del objeto mismo de carácter sensible. Justamente, esta es la dirección que tomarán las presentes consideraciones.

\section{LA MANIFESTACIÓN DEL OBJETO SENSIBLE}

En las investigaciones fenomenológicas es conocida la caracterización husserliana de la percepción táctil: "La misma sensación táctil, concebida como rasgo del objeto 'exterior' y concebida como sensación del cuerpo-objeto." (Husserl, 1952, p. 147). La sensación táctil presentaría para Husserl una así llamada "sensación doble" (Doppelempfindung), la cual advierte que cuando el dedo toca una superficie, a su vez, éste también es sentido o "se toca”. En el mismo sentido, Katz (1925, p. 17-21) reconoce que las sensaciones del tacto presentan el rasgo de una "bipolaridad" (Bipolarität). ${ }^{12}$ Por una parte, cuando tocamos con la palma de la mano una superficie áspera, distinguimos que la aspereza pertenece al objeto tocado, mientras que entendemos que la sensación de cosquilleo en la piel no es sino un dato perceptivo de nuestra mano, es decir, es una sensación que nos incumbe sólo a nosotros. Precisamente, un ámbito

\footnotetext{
${ }^{12}$ La relación entre Katz y Husserl, sin embargo, no es explícita. En la obra que acá mencionamos, Der Aufbau der Tastwelt, no hay referencia a éste. Cabe destacar, empero, aquella dirección en su investigación sobre el tacto que testimonia su acercamiento a las descripciones de la percepción husserlianas. Por una parte, Katz (1925, p. 246) advierte que la teoría psicológica no puede anteponerse y prescindir de "[...] puntos de vista fenomenológicos puros.". Ciertamente, éste comprende tal investigación como una descripción inmanente de las vivencias táctiles, y su obra de carácter psicológico experimental rinde cuenta del modo como ellas donan al objeto. Es así como, subrayando las "maneras de aparecer" o "variantes fenoménicas" (Erscheinungsweise) implicadas en este tipo de donación, Katz (1925, p. 25) destaca la relevancia de los "factores psíquicos" en la construcción del mundo táctil. En este contexto, es importante observar además que Katz, en su intento de mostrar la pertenencia de los datos táctiles a la donación objetual, recurre a aquellas fenomenologías de la percepción como las desarrolladas por Hofmann (KATZ, 1925, p. 10) y Schapp (KATZ, 1925, p. 20 y 94), citadas también en el presente trabajo.
} 
de la sensibilidad como lo táctil parece mostrarnos, según la terminología de Katz (1925, p. 19), un polo eminentemente objetivo y otro de carácter subjetivo. De hecho, muchas veces, es la dimensión subjetiva la que se acentúa en la experiencia del tocar, sin que lo que sintamos proporcione dato alguno sobre el objeto. Si con la punta de los dedos rozáramos con fuerza y por un tiempo prolongado una superficie lisa, no se tardará en sentir una sensación de calor que podría convertirse, finalmente, en ardor. En tales circunstancias, la textura de la mesa permanecería completamente muda. Mas, si se desplazaran suavemente los dedos sobre ésta, la dimensión subjetiva parece reducirse atencionalmente, incluso, desaparecer, predominando la lisura de la superficie como carácter objetivo de la mesa en cuestión.

Algo análogo ocurre en el ámbito del gusto. Dependiendo de su intensidad, puede acentuarse tanto la dimensión objetiva como subjetiva. Es el caso de lo extremadamente salado, que oculta el sabor de lo que comemos, es decir, los caracteres del objeto. Otras veces, el polo objetivo gana primacía, como cuando el experto es capaz de identificar en el vino aspectos frutales o herbales, describiéndolos como características de lo degustado mismo. Por su parte, los olores también parecen oscilar en tal duplicidad. El aroma que advertimos en un alimento es un índice para decidir si éste puede o no ser comestible. Es decir, el olor nos puede hablar del objeto mismo. Mas, cuando nos confrontamos con aromas altamente penetrantes, como el vinagre, la intensidad puede ser tal que nada nos dice de las cosas mismas. En este sentido, Hans Henning, en su trabajo El olor (1916), puede identificar entre un "olor-circunstancia" (Gegebenheitsgeruch) (HENNING, 1916, p. 30), según el cual se entiende que el olor mismo es el todo de nuestra experiencia, sin que se pueda identificar la ubicación del objeto, y un "olor del objeto" (Gegenstandsgeruch) (HENNIG, 1916, p. 31), siendo aquel que proporciona efectivamente las propiedades de algo. ${ }^{13}$ Cabe señalar, sin embargo, que el olor por sí mismo no satisface por completo la donación del objeto. Como Henning mismo advierte, éste requiere de una ayuda visual, como cuando en una habitación sentimos olor a flores y es luego, visualmente, cuando detectamos que se trata de aquellas (HENNING, 1916, p. 31).

\footnotetext{
${ }^{13} \mathrm{La}$ inclusión del psicólogo Hans Henning en el círculo fenomenológico descrito atiende a que su libro Der Geruch resulta ser una fuente de sugerentes descripciones respecto del rol presentativo de los olores. Destacando tales descripciones es que Cassirer (1982, p. 150), por ejemplo, realiza una revisión de las consideraciones fenomenológicas que rinden cuenta de la constitución sensible objetual, refiriendo a trabajos como los de Henning, respecto de los olores, de Schapp, respecto de los colores, y de Katz, en relación a la donación táctil.
} 
Ahora bien, el caso de la vista y el oído son diferentes. Como advierte Husserl (1952, p. 147), la percepción visual no presenta el aspecto subjetivo propio de las sensaciones táctiles, de los olores y sabores. Por su parte, el ojo no se ve a sí mismo cuando miramos un objeto. De igual manera, no escuchamos el oído cuando se escucha un sonido. Sin embargo, y esto es lo decisivo, la carencia del polo subjetivo del oído y la vista advierte acerca de un rasgo importante de las sensaciones mismas: ellas parecen poseer, como carácter sine qua non: la presentación del objeto exterior, ya sea con o sin referencia a una dimensión eventualmente subjetiva. La percepción sensible es, se puede afirmar, ante nada, presentadora de las cosas con las cuales nos relacionamos. Sin tal condición, sin sensibilidad, algo así como "cosas corrientes" sería impensable. Justamente, será por la vía de este rol eminentemente "presentativo", propio de todas las cualidades referidas, que quisiéramos interrogar la naturaleza del objeto sensible.

A este propósito contribuye en gran medida tomar en cuenta algunas consideraciones de Wilhelm Schapp. Se trata de su tesis doctoral, Aportes para una fenomenología de la percepción (1910), redactada bajo la supervisión del propio Husserl. En estos análisis, se muestra cómo es que colores, tonos o texturas, no son ellos mismos la cosa percibida, sino que son aquellas instancias necesarias para su exposición. Al observar, por ejemplo, una hoja de papel, ésta se presenta desde diversos matices del blanco, con sectores más sombríos o más luminosos que otros. Asimismo, al tocarla, la hoja es percibida con cierta lisura, aunque no del todo constante. Sin embargo, nada más lejos del fenómeno perceptivo resultaría afirmar que la experiencia de la hoja radica en la percepción de blancos, claroscuros o lisura. Más bien, se percibe, ante nada, una "hoja" que no es uniformemente blanca, con una lisura no del todo constante. En este contexto, Schapp (2013, p. 46) advertirá que los aspectos sensibles corresponden a lo "presentante" (Darstellendes), mientras que lo percibido propiamente tal sería lo "presentado" (Dargestelltes) (SCHAPP, 2013, p. 47): la "cosa" en cuanto tal.

Ciertamente, tomada de Husserl, ${ }^{14}$ esta distinción desarrollada por

\footnotetext{
${ }^{14}$ Husserl (1973, p. 50) advertirá: "Cuando seguimos el contenido de la sensación paso a paso, con evidencia hallamos como algo necesariamente perteneciente a él, que cada uno de sus momentos posee una especial función expositiva (Darstellungsfunktion) que le pertenece." Sin embargo, esta donación sensible no rendiría cuenta total de la cosa dada, pues, por ejemplo, al percibir su parte delantera, tenemos conciencia, mas no percepción, de la parte trasera. Siendo nuestra intención destacar el rol expositivo de las cualidades sensibles como constitutivo la donación objetual, hacemos énfasis en los análisis de Schapp que sólo enfatizan tal aspecto. No obstante, al igual que Husserl (SCHAPP, 1976, p. 9), su interés es más amplio: “[...] desde la mirada al objeto - dirá éste - regresar al mirar mismo, y
} 
Schapp es sumamente conveniente en cuanto permite ordenar las dimensiones del aparecimiento sensible de un objeto. En primera instancia, podríamos hablar de la percepción de un espacio en general. No es difícil advertir que las cualidades visuales exponen espacio. La extensión completa de un paisaje sólo es percibida mediante los colores que la presentan. Igualmente, los sonidos también parecen donar un espacio. El ruido de la vajilla, en efecto, que se escucha al otro lado de la pared abre espacios determinados (SCHAPP, 2013, p. 27). Si bien es cierto, Schapp entiende la esencia del espacio como autónoma respecto de las cualidades sensibles, hará énfasis en que su aparición corriente depende de este "ropaje" (Umkleidung) (SCHAPP, 2013, p. 43) de carácter sensible.

Así es como, aproximándonos aún más a la constitución del objeto mismo, se puede afirmar que la cualidad sensible presenta también sus dimensiones particulares. En cuanto a la visión, el color acompaña en cada momento las dimensiones de lo observado. Por otro lado, el sonido también habla de las dimensiones de los cuerpos, como cuando se escuchan las diferencias de tamaño de los vehículos que transitan por una calle aledańa. En el caso del ámbito táctil, la percepción del espacio puede advertirse aún sin problemas. Y esto no sólo si se desliza la mano a lo largo de una superficie. Si se cubre un libro con un trozo de género, las texturas presentan la rugosidad del género, no obstante, tocamos, a través del mismo, el objeto cubierto, advirtiendo sus propias dimensiones, esto es, la "estructura de su superficie" (KATZ, 1925, p. 31). Tales vivencias, comenta el mismo Katz, serían comunes en la praxis médica (1925, p. 103). El médico trata con el cuerpo del paciente con sus guantes de goma, y, aunque la percepción de las texturas queda inhabilitada, palpando, éste puede enterarse sin dificultades del tamaño y estado de los órganos internos que examina.

Así, los índices dados en relación tanto al espacio en general como a las dimensiones específicas de las cosas realzan una diferencia decisiva en el ámbito sensitivo. En tanto que expositivas, las cualidades sensibles han de distinguirse de las propiedades que pertenecen más bien a la cosa expuesta. La dimensión espacial del objeto, por ejemplo, ya no puede ser considerada como un dato sensible. En estricto rigor, ella se presenta mediante este ropaje visual, táctil y auditivo. Lo mismo ocurre con la dureza o blandura, la flexibilidad o solidez, el peso o la liviandad. Todas estas propiedades pueden

lo que es más significativo aún, a todo aquello que en tal mirar aparece en el pensar-sobre, en un estar ocupado con." 
ser consideradas como constitutivas de las mismas cosas, empero, es posible distinguir sin problemas su naturaleza eminentemente objetual de aquella que corresponde a los datos sensibles. Como se advierte, el así entendido "ropaje" resulta ser el cimiento último de la aparición. Tomando en cuenta lo anterior, lejos estaríamos ya de entender las cualidades sensibles como meros estados nerviosos en el sujeto. Mientras esta interpretación arroja a lo sensible a la externalidad de una instancia material, los nervios, ella sesga, a la vez, su rol primario de exhibidor de las cosas del mundo. Mas, en razón de esta condición presentativa, afirmamos, es que las cosas no se componen precisamente de asperezas, sonidos, sabores, colores, luminosidades, sino, en estricto rigor, de propiedades cósicas como peso, consistencia, elasticidad, etc., exhibiéndose desde las cualidades sensibles. Hofmann dirá, en este sentido constitutivo de la objetualidad del objeto: "La meta de nuestras percepciones es, no obstante, la mayoría de las veces, la cosa con sus propiedades [...]." (HOFMANN, 1913, p. 87). En definitiva, el mundo propio de la sensibilidad muestra su auténtica primacía para la vida corriente: se trata de aquella esfera omnipresente, el hogar en el que nos guarecemos, cuando vivimos corrientemente en un mundo cotidiano y concreto.

Sin embargo, sería importante prestar atención a un atributo cósico particularmente problemático cuando la aparición del objeto cotidiano se entiende en esta relación de dependencia con la sensibilidad: la figura. En directa relación con las dimensiones mismas del objeto, la figura, en el ámbito cotidiano, esto es, sensible, es completamente variable. Dicha propiedad parece asociarse tanto a la vista como al tacto, ya que ruidos, olores y sabores no exponen tal propiedad. Nos inclinamos ante una mesa, y la superficie se expande o contrae según la perspectiva desde la que la miremos. Es Oskar Becker quien, por ejemplo, acentúa la preeminencia de la "cosa visual” (Sehding) o "squiágrafo" (BECKER, 1973, p. 72) en un estadio fundante del espacio homogéneo y continuo euclideano. Es propio, podemos decir con él, en el nivel sensible tal aparición "perspectivística" del objeto percibido. En dicha esfera, las figuras presentan una constante y característica "deformación”. ${ }^{15}$

Mientras tanto, en el ámbito de lo táctil, la figura sólo es aprehendida parcialmente. Y, por lo demás, a pesar de que lo táctil parezca primar por sobre los otros sentidos, al implicar un contacto directo con el objeto, no garantiza

${ }^{15}$ En este punto es importante tener en cuenta las consideraciones sobre el cuerpo vivo (Leib) husserlianas, y cómo éste determina una orientación primaria en el espacio en tanto que "punto cero" (Nullpunkt), siendo determinante en la perspectiva espacial de la percepción cotidiana (HUSSERL, 1952, p. 158 s.). 
en todos los casos una percepción a la que podamos darle crédito absoluto. ${ }^{16}$ En efecto, lo cierto es que la percepción táctil puede corregir la percepción visual (KATZ, 1925, p. 41-42). Ella exhibiría auténticas discontinuidades al tocar la arena, por ejemplo, mientras que visualmente percibimos sólo un continuo. Pero, cuando acariciamos un tejido de encaje, corregimos con la vista la discontinuidad efectiva de la superficie que percibimos táctilmente como una rugosidad permanente. Así, no hay una razón suficiente para afirmar que el ámbito de lo táctil sea el auténtico lugar de la figura del objeto. Por lo demás, éste también es limitado, y hay figuras a las que jamás tendremos acceso mediante él, como es el caso de la luna, percibida y concebida siempre visualmente como un disco (HOFMANN, 1913, p. 74 s.).

En este contexto, surgen interrogantes sugerentes al momento de abordar el problema de la figura. Por una parte, al alejarnos de un objeto rectangular, la agudeza de sus esquinas parece atenuarse, hasta que, a determinada distancia, desaparecen sus cantos. Hofmann destaca que, a pesar de tales diferencias perceptivas, tomamos una de las diversas percepciones como aquella que nos indicaría la "figura visual natural" (natürliche Sehgestalt) (HOFMANN, 1913, p. 74) del objeto. La pregunta evidente es, por tanto, por aquel criterio según el cual una de tales percepciones gana el rango de veracidad respecto de cualquier otra. Schapp, por su parte, ilustra el problema mediante una ilusión. Vemos tirado en el piso un pedazo de greda, el que, tras una mirada atenta, resulta ser, más bien, un trozo de tocino. ¿Qué es lo que permanece idéntico en tal transformación (SCHAPP, 2013, p. 100)? Tales advertencias conducen, por cierto, a una pregunta fundamental respecto de la percepción sensible, a saber: ¿cuál es la relación entre identidad y sensibilidad? En concreto, ¿habita la identidad del objeto percibido en las cualidades sensibles mismas?

Es claro que las diversas cualidades sensibles nos presentan un mismo mundo exterior. Cada cualidad sensible nos ofrece una vía para llegar siempre a lo mismo. El daltónico (KATZ, 1925, p. 33) o el sordo, quizás no puedan acceder a ciertos caracteres objetuales mediante el color o el sonido, no obstante, siempre

\footnotetext{
${ }^{16}$ Cabe distinguir estas discusiones sobre el tacto de la eventual primacía atribuida por Aristóteles al mismo. En De anima se lee: "Igualmente, sin el tacto no se da ninguna de las restantes sensaciones, mientras que el tacto sí que se da sin que se den las demás" (ARISTÓTELES, De anima 415a3-4). Se advierte que la primacía en juego en este caso es "genética". Aristóteles entiende lo táctil como una sensación básica que sería requerida por todo otro sentido, suponiendo que cada sensación, ya sea, gustativa, olfativa o visual, implica, en sentido amplio, un cierto "contacto". Por nuestra parte, destacamos que esto no conduce a asumir su superioridad "cognitiva", pues hay casos en los que se puede acceder mejor a las propiedades del objeto mediante otros sentidos que mediante el sentido en cuestión.
} 
pueden encontrar otra vía para acceder a las mismas propiedades cósicas que un sujeto normal pudiera advertir. Se trata de la posibilidad, por ejemplo, de acceder a propiedades sonoras mediante las vibraciones, como ocurre, dicho sea de paso, en el famoso caso de Hellen Keller. Katz (1925, p. 192) destaca aquellos relatos donde ella, en el intento de tratar con su sordera, manifiesta poder sentir la melodía o el propio canto al palpar un piano o el sector de la laringe de un cantante. Para Katz, dicha capacidad de acceso al mundo auditivo es posible mediante los datos vibratorios pertenecientes a lo táctil. En efecto, estos son entendidos por él como una dimensión que, a diferencia de la cercanía de la presión táctil, puede hacer del tacto un "sentido de lejanía" (Fernsinn) (KATZ, 1925, p. 211).

Así, no es que percibamos un mundo visual y otro auditivo, sino que, como advierte Schapp (2013, p. 32) se trata de "[...] un mundo que aparece en colores y sonidos." Es cierto, por lo demás, que cada cualidad sensible es intransferible a otra respecto de su contenido, pero, pese a ello, todas éstas presentan e instalan a la vida corriente en una misma realidad. Cabe preguntarnos, por tanto, si esta mismidad del mundo habita en los múltiples contenidos sensibles o, tal vez, en el objeto total. Es decir, ¿qué es lo que hace, en última instancia, que todas las características de un objeto se reúnan en una misma cosa?

Pues bien, la relación de íntima dependencia de las propiedades cósicas con el carácter "presentativo" de las cualidades sensibles conduce al problema de la identidad última de la cosa sensible. Sin embargo, la dificultad de esbozar alguna solución es que se advierten en la cosa presentada diversas propiedades objetuales como solidez, resistencia, elasticidad, flexibilidad, pero que al momento de explorar dónde habita su eventual unidad, no hallamos entre ellas alguna que parezca cumplir tal función. Por lo pronto, la figura del objeto es la propiedad que más relación pareciera guardar con la identidad. Desde una mirada ingenua, ésta se entiende como lo invariable de las cosas. Y, sin embargo, un examen del ámbito cotidiano de la sensibilidad nos advierte de su constante variabilidad. De esta manera es que nos vemos conducidos al problema de la identidad del objeto sensible. A nuestro juicio, algunas consideraciones sobre dicho problema podrían ayudar a esbozar una caracterización del aparecer del mundo sensible en cuanto tal.

\section{LA EXTERIORIDAD DE LAS COSAS: EL CARÁCTER SITUACIONAL DE LA SENSIBILIDAD}

El supuesto de la substancia podría llevarnos a responder a la pregunta por la identidad de la cosa sensible reparando en su constitución interna. Se 
podría asumir que su mismidad, i.e., lo que permanece idéntico en el cambio, es alguna dimensión constitutiva subyacente, invariable en relación a ella misma. Sin embargo, esto supondría que ésta sería, en esencia, en si y para sí. En tales análisis, por lo demás, la sensibilidad requeriría ser entendida como una relación unilineal entre el sujeto y un objeto aislado. Cada acto perceptivo consistiría en la aprehensión de esta o aquella cosa que se presenta, de forma separada. Pero, no todo lo que se exhibe en la percepción parece mostrarse así. Es posible mirar un libro entre muchos en una estantería. Mientras lo hacemos, podemos "acentuar" el libro visualmente respecto del todo en el que se encuentra, según lo que Schapp (2013, p. 68), por ejemplo, entiende como un "mentar que se encuentra entresacando" (herausgreifend Meinen), para luego "empacarlo" (Anpacken) (SCHAPP, 2013, p. 68) atencionalmente en el centro del campo visual. No obstante, en tal acto, el libro no aparece como un objeto aislado, sino dado en un trasfondo. Y, aunque desdibujado, dicho trasfondo está siempre en potencia de ser atendido como parte de la experiencia del libro.

Ahora bien, lo anterior no se corresponde con otro modo de ejecutar la visión. Podríamos detenernos nuevamente en el libro, con el fin de detectar en su superficie imperfecciones, rasguños, etc. A diferencia del anterior, en este modo de percepción el objeto conforma el todo, y mientras atendemos a sus partes o sectores, son estos los que se constituyen en la totalidad perceptiva, mientras que lo restante no queda como fondo difuso, sino que palidece y desaparece. En este último caso se trata de un tipo de percepción visual que "detecta propiedades", que las examina, las aísla y las describe. Precisamente, este tipo de observación es el que pareciera servir de modelo cuando se considera que la percepción presenta objetos aislados. A diferencia de una observación de carácter cotidiano, como la descrita anteriormente, esta por Schapp (2013, p. 65) llamada "percepción observante" (beobachtende Wahrnehmung) separa lo observado de su contexto perceptual y lo presenta como una totalidad. Justamente, en el marco de este acto perceptivo se justifica la exigencia de la veracidad de la recepción de la realidad. A juicio de Schapp (2013, p. 69), “[...] este tipo de percepción parece proporcionarnos auténticamente el conocimiento de la cosa." En efecto, tal modo de observar correspondería al que es propio del científico. El científico no mira, de hecho, sino buscando información clara, distinta y verdadera de lo que observa. Y es que no habría observación científica si no se cumplieran tales requisitos. Se detecta, así, en este modo de percepción una disposición más bien teórica, distante, por lo demás, de la manera como percibimos cotidianamente. 
Pero cabe preguntarnos cómo es que el mundo sensible aparece en una percepción de carácter cotidiano, al margen de la concepción substancial de la realidad, de su primacía epistémica y de la requerida actitud teórica que ha de poseer su observación. En primera instancia, se puede afirmar que no toda percepción se realiza como un examen acucioso, con el fin de obtener características claras y distintas de las cosas. Cuando hallamos con la mirada el lápiz que usaremos para firmar, basta con ver su localización así como algún par de características vagas que indiquen que tal es el lápiz que utilizaremos. Tal mirada carece de todo tipo de "claridad y distinción". Lo exhibido en la inmediatez sensible no posee caracteres epistémicos, no expone datos "objetivos" para rendir informes sobre ellos, sino, ante nada, se manifiesta en cuanto una posibilidad inmediata de "trato".

Como se advierte, ambos modos perceptivos son muy distintos. En uno, requerimos del objeto claridad y distinción. En el otro, el mundo se nos presenta con cierta imprecisión para habitar entre las cosas. En este último, simple y fundamentalmente somos instalados en el mundo. Cuando vemos una arańa, aunque luego resulte ser una pelusa movida por una corriente de aire, no "creemos" verla. En ese instante estamos efectivamente con la arańa, sorprendidos o asustados. Es este ya destacado carácter expositivo de la sensibilidad el que nos relaciona con un entorno. ${ }^{17}$ Así, en un nivel inmediato, no se nos dona un mundo para conocer, sino para vivir. El hecho de que las cosas en tal nivel no se presenten claras y distintas no implica, entonces, un aparecer "deficiente". Su efectividad se acredita con todo rigor cuando advertimos que en este modo de aparición es cuando nos instalamos de lleno en nuestro entorno inmediato. Así, el mundo sensible aparece, aunque no necesariamente como objeto, sino como el primer suelo, pre-temático, de relaciones cotidianas.

Precisamente, es en este punto donde se advierten las ganancias que se obtienen recurriendo al círculo de fenomenólogos tempranos. Suscribiendo

\footnotetext{
${ }^{17}$ En este sentido, seguimos a Lambert Wiesing, quien indica que lo que la percepción proporciona como nuestro mundo perceptivo aparecería en la forma de "algo es el caso" "[...] dass, etwas der Fall ist" (WIESING, 2009, p. 140). Cuando vemos un remo quebrado en el agua, vemos la quebradura como que "ese sería el caso", mas esto no implicaría un conocimiento. De hecho, podemos saber que el remo no está quebrado y, sin embargo, seguir viéndolo como "si fuera el caso" de que lo estuviese. Esto lleva a distinguir y desvincular lo que aparece perceptivamente de aquel mundo que construimos cognitivamente y, así, detectar niveles diferentes de su aparecimiento. Según Wiesing: "[...] la percepción de un remo quebrado no proporciona ningún saber falso, porque una percepción no proporciona en absoluto un saber, y no se encuentra necesariamente vinculada a una convicción" (WIESING, 2009, p. 141).
} 
tesis fenomenológicas de la percepción, en un afán por desvincular la investigación psicológica de consideraciones fisiológicas, y tomando el problema de la percepción desde un sentido eminentemente constitutivo del objeto percibido, los trabajos de Schapp, Hofmann, Katz, Henning o Becker, proporcionan descripciones precisas acerca de la constitución cotidiana del objeto percibido en el nivel de la vida corriente. Es la constitución del objeto cotidiano la perspectiva en la cual las cualidades sensibles acusan ser más que una mera capa adherida a los objetos y se realzan como el modo fundamental de su manifestación. La riqueza de tales descripciones muestra sistemáticamente cómo en cada uno de los sentidos, las cualidades sensibles a cada uno de ellos pertenecientes, son un modo de manifestación, ya sea del objeto visto, del objeto oído, degustado, etc. Cabe señalar que estos trabajos son una fuente valiosa para emprender posteriores consideraciones en el contexto de una fenomenología de los sentidos (DIACONU, 2013). Son ellas mismas, sustentadas en un suelo de experiencias concretas, las que plantean el problema de la identidad misma del mundo cotidiano, primariamente exhibido mediante las cualidades sensibles. Como se advierte, la principal ganancia de las descripciones fenomenológicas acá comentadas es, por ende, de naturaleza metódica. Se trata de afianzar el modo adecuado de realzar el mundo sensible en su exterioridad e identidad primarias.

En el intento de indagar en dicho problema, se puede recurrir nuevamente al trabajo de estos autores para examinar hacia donde parecen dirigirse las consideraciones fenomenológicas emprendidas. Se trata de preguntarnos por qué es que, a pesar de que cuando "objetivamente" debiéramos estar viendo, escuchando o tocando un objeto, resulta que no lo percibimos. Es el caso, por ejemplo, de lo que la psicología denominó "petite perceptions", es decir, percepciones vagas que no constituyen el contenido de la percepción sensible consciente. Según el ejemplo de Hofmann (1913, p. 50), se trataría del tic-tac del reloj, el cual no necesariamente es escuchado, a pesar de que constantemente "suene" o cuando apoyamos el brazo sobre la mesa, sin que, por ello, percibamos en cada momento la superficie de la misma. En tales casos, la exterioridad del mundo parece enmudecerse. Esto, a nuestro juicio, indica un carácter importante de su modo propio de aparición. En efecto, el tic-tac del reloj no se exterioriza mientras estamos instalados en la situación del leer. La mesa no es parte, por ejemplo, del ejercicio de atender a aquel con quien conversamos. Más bien, la exterioridad del mundo se enfatiza en peculiares direcciones, justo cuando nos hallamos propiamente en "nuestros asuntos". Atendiendo a la esencia de las cualidades sensibles, i.e., 
a su carácter presentativo, el mundo exterior parece ser primariamente un para nosotros, y jamás un para sí, neutro e inerte. En otras palabras, la exterioridad sensible parece ser tal, en la medida en que se integra en una determinada situación, es decir, la exterioridad de la cosa vendría a acaecer siempre en nuestra situación particular.

Es importante tener en cuenta, empero, que recurrir a una "situación" como ámbito de aparición del mundo exterior es un paso que los autores comentados no dan explícitamente. Será el énfasis existencial heideggeriano, ajeno, por supuesto, a la época de sus reflexiones, el que instalará la relación fáctica como ámbito de apertura del mundo en la discusión fenomenológica. Un claro ejemplo de este énfasis en la consideración de la sensibilidad en un nivel existencial es Wilhelm Schapp. Mientras que en su tesis doctoral éste realiza descripciones directamente influidas por Husserl, cuatro décadas más tarde, es Sein und Zeit de Heidegger la obra que guiará sus análisis. Resulta interesante dicho giro en sus descripciones para lo que quisiéramos discutir.

En efecto, en su obra In Geschichten verstrickt, Schapp (1953) advierte que conceptos como "color", "textura", e incluso aquellos con mayor pretensión de concretud, como "dureza", "elasticidad" o "resistencia" han de ser ahora entendidos en relaciones de trato, ya que tales nociones parecieran transgredir la presentación cotidiana de lo sensible. Y es que más bien hallamos, como éste advierte ahora en términos existenciales, la materialidad de las cosas en el sentido en que son "fraguables" o "martillables", "recortables", en un determinado "trato" (Hantieren) (SCHAPP, 1985, p. 21). En efecto, no oímos meros "sonidos", sino melodías agradables o extrañas, murmullos o pasos de un conocido o un desconocido. Una formulación cuidadosa de la sensibilidad cotidiana, en este sentido, no podría recurrir a conceptos como "tacto", "olfato", "oír", etc. En la esfera sensible inmediata no realizamos el acto absoluto de tocar, sino más bien, acariciamos, empujamos, siempre "algo". "Tocar", señala Schapp (1985, p. 24), se trata primariamente de "presionar" o "deslizarse".

Justamente es este giro fáctico el que demarca otro modo de indagar en el fenómeno de la percepción. Es desde él que una relación de trato como ámbito de aparecimiento de las cosas del mundo se puede entender situacionalmente. Así, el caso de las petite perceptions no podría entenderse como si un "mundo objetivo" proyectase todas las cualidades sensibles posibles, y que ellas estuviesen dispuestas a ser "seleccionadas" atencionalmente por nosotros. Atendiendo a una relación fáctica de trato, parece ser inapropiado hablar de 
una eventual "discriminación" de las cualidades que nos interesan. Desde las consideraciones de Schapp, parece ser que es instalados en una situación de trato que lo presentado aparece, y esto, podríamos agregar, siempre según un realce sensible de las cosas. En dicho trato, por ejemplo, nos es indiferente el color del revestimiento del lápiz grafito, en tanto que la primacía la tiene el trazo mismo del grafito que se quiere conseguir para un determinado dibujo. En este sentido, se podría hablar de una "perspectiva sensible" de carácter situacional, en la cual habitamos y que se constituye al interior de determinados tratos con las cosas. Se advierte, así, que la exterioridad de las cosas acaecería en una determinada perspectiva para nosotros. No se trataría, por cierto, de una perspectiva local, sino, más bien, de una situacional.

En consecuencia, según una dirección determinada y en un énfasis específico parece constituirse el todo de nuestra experiencia, es decir, el ámbito "con lo que contamos". Es, en efecto, desde estas discusiones que obtenemos una caracterización del modo de aparecimiento del mundo sensible en cuanto ámbito inmediato para la vida concreta. Dicho mundo es tal, afirmamos, en una presentación enfatizada sensiblemente. Dichos "énfasis sensibles" indican que la aparición de las cosas exteriores no puede ser concebida como si se tratase de objetos aislados, sino que, siguiendo el ejemplo de una percepción cotidiana como la de observar un libro entre muchos otros en una estantería, tal aparecer radicaría en la donación de un "mundo enfático" al interior de una relación fáctica, donde, instalados en ella, habitamos arrojados "entre múltiples cosas", ocupándonos con ellas de algún modo específico. Por ello, nuestro mundo exterior inmediato parece presentarse, ante todo, como un entramado sensible, donde "sensible" querría decir: aquello que nos "aqueja", en la medida en que nos abre a relaciones fácticas concretas.

A nuestro parecer, es en este contexto donde puede esbozarse una respuesta en torno a la identidad, a la mismidad del mundo exterior. Se puede decir, en efecto, que aquel mundo que resulta ser el inmediato para la vida, aquel cuya presentación depende de la sensibilidad, gana su identidad al interior de dicha relación situacional, es decir, en medio de posibilidades de trato en las que nos hallamos arrojados. En dicho todo situacional descubriríamos y nos atendríamos siempre al mismo mundo, mas viviendo en la constante variación de su manifestación. ${ }^{18}$ Dicho de otra manera, la mismidad del mundo exterior

\footnotetext{
${ }^{18}$ Es importante mencionar que el concepto de "situación" al que se alude en estas discusiones posee una íntima relación con la noción de "mundo" o "espacio", realzadas por Merleau-Ponty en sus sentidos "vitales". Por una parte, en Phénoménologie de la perception se caracteriza al espacio como "[...] el modo por el cual la posición de las cosas se vuelve posible” (MERLEAU-PONTY, 2005, p. 290), mientras
} 
pareciera radicar en aquel para quien hay mundo, en tanto que ocupado con él, esto es, en nosotros, mas no en la estructura retraída de una substancia. La identidad del mundo inmediato acaecería, por tanto, en un horizonte de ocupaciones en las que nos atenemos.

Así es como estas discusiones conducen a realzar nuestra situación como el ámbito en el que el mundo sensible se enfatiza y gana su propia unidad. Es en ella, pues, donde la cosa sensible adquiriría su particular unidad y su propia exterioridad. Se advierte, entonces, que el problema del mundo exterior es, finalmente, un problema que refiere a nosotros, como quienes le otorgan su propia exterioridad e identidad. Nosotros parecemos ser los donadores del suelo más concreto en el que vivimos, y dicho suelo, las cosas entre las que habitamos, nos serían sensiblemente concretas en la medida en que nos arrastran para sí al trato, mientras habitamos nuestra situación. El carácter de lo sensible es tal, podríamos afirmar, en tanto que "se vuelve" asunto nuestro, i.e., se integra en nuestro "ámbito vital", como el todo que asumimos en cuanto nuestro lugar vital específico. Justamente, en tal sentido es que el mundo sensible se nos aparece: en tanto que lo que contamos para poder vivir. ${ }^{19}$

Pues bien, acabando con estas discusiones, quisiéramos una vez más destacar cuán fructífero resulta, entonces, volver a aquellas obras fenomenológicas tempranas al momento de abordar la percepción sensible. Y es que desde ellas se tiene a disposición un material de consideraciones fenomenológicas detalladas que abren un camino hacia una comprensión del mundo en la intimidad de una relación con la propia vida humana. Al respecto, e integrando el carácter situacional de la donación del mundo sensible, es que podemos afirmar que, más allá de que la exterioridad de nuestro mundo sea concebida en un sentido "objetual” y, por ende, teórico y ajeno a su auténtica inmediatez, éste parece

que en su presentación de 1946, Le primat de la perception et ses conséquences philosophiques, éste se refiere a mundo como "[...] el estilo universal de toda percepción sensible" (MERLEAU-PONTY, 1996, p. 50). En ambos casos, el fenómeno a realzar es el de un horizonte de manifestación de las cosas que aparecen, el mismo que ha de entenderse como aquel sentido rector y directriz según el cual se configura su concretud inmediata, precisamente, podríamos agregar, con todas sus variaciones concretas. En esta dirección es que queremos destacar el carácter situacional del aparecimiento del mundo sensible, haciendo énfasis en que las así llamadas "cualidades sensibles" serían, entonces, la concreción específica que aparece a la vida inmediata de aquel horizonte de manifestación que Merleau-Ponty tan oportunamente expone en cuanto un "modo" o "estilo".

${ }^{19}$ Desde esta perspectiva queremos entender una breve precisión de lo "sensible" que Heidegger hiciera a propósito de Nietzsche. Respecto de la problemática del cuerpo, Heidegger dice que cuerpo ha de ser "sensible" (sinnlich), en la medida en que "[...] se precipita hacia algo, para incluirlo en su ámbito como consistente" (HEIDEGGER, 1989, p. 169). 
manifestarse "sensiblemente" en cuanto insta a una relación de trato. Con ello se hace posible advertir que la concretud sensible del mundo exterior radica en una exhortación a vivir tratando con las cosas, y no en una mera objetualidad dispuesta a ser conocida. ${ }^{20}$ Por esta vía, se hace evidente que las consideraciones fenomenológicas expuestas nos conducen necesariamente a "nosotros", es decir, a la vida humana misma, como el contexto en el cual se despliega y, finalmente, "hay" mundo sensible. Con las descripciones emprendidas tempranamente en la fenomenología, en fin, se hace posible no sólo afianzar metódicamente un estudio de la percepción cotidiana, sino que además, la riqueza de tales análisis permite indagar en los niveles de constitución de lo sensible con los detalles necesarios como para no traicionar el fenómeno en juego: la aparición del mundo de carácter cotidiano. A nuestro juicio, y como fue el esfuerzo desde los inicios de la fenomenología, su aclaración corresponde a un paso propedéutico necesario en vistas, ante todo, a una aclaración de aquella relación vital que parece acontecer necesariamente entre la vida humana y aquel medio vital en el que ella habita "entre sus propias cosas".

JOHNSON, F. "Among daily things": early phenomenology and the appearance of the sensible world. Trans/form/ação, Marília, v. 41, n. 1, p. 163-190, Jan./Mar., 2018.

\begin{abstract}
This paper aims to describe the mode of appearance of the sensible world, understood as the first basis on which human life is effectively placed among concrete things. We will try to distinguish the level of immediate daily presence of the outside world from the way the outside world has traditionally been conceived, namely, as a mere "objective" manifestation. In this context, we will draw upon phenomenological descriptions of perception which appear mainly in philosophical and psychological works of early 20th century, and which were influenced by Edmund Husserl's studies on perception. On this basis, we inquire how the sensible world appears in daily life without the character of "epistemic truth". Our purpose thus is to understand the outside world in an intimate relationship with a vital situation in which life itself opens its own basis for living.
\end{abstract}

KeYwords: Perception, Sensory quality, Outside world, Everyday life, Early phenomenology.

${ }^{20}$ Fink distinguirá claramente entre "aparecer" (Erscheinen) y "volverse-un-objeto" (Zum-Gegenstandwerden) (FINK, 1990, p. 33), como modos distintos según los cuales el ente puede donársenos. A nuestro juicio, las consideraciones sobre la manifestación sensible del mundo deben abordar el problema de las cualidades sensibles en aquel nivel inmediato, en el cual el existir se encuentra en una relación de trato fáctico con su mundo, mas donde no acaece aún una tematización u objetualización explícita del mismo. Nos referimos, pues, a la esfera pre-temática (vor-thematisch) desde la cual Heidegger explicitaría el modo de ser del mundo circundante (Umwelt) en cuanto "ser-a-la-mano" (Zuhandenheit) (HEIDEGGER, 2001, p. 69) y que el mismo Fink pareciera tener en vista en su propia distinción. 


\section{REFERENCIAS}

ARISTÓTELES. Acerca del alma. Madrid: Gredos, 1978.

BECKER, O. Beiträge zur phänomenologischen Begründung der Geometrie und ihrer physikalischen Anwendung. Tübingen: Max Niemeyer, 1973.

CASSIRER, E. Philosophie der symbolischen Formen III. Darmstadt: Wissenschaftliche Buchgesellschaft, 1982.

TOMÁS DE AQUINO. Suma teológica. V. I / Parte 1. Madrid: BAC, 2001.

DESCARTES, R. Principes de la philosophie. Oenvres de Descartes. Paris: Vrin, 1996a. V. IX. . Les méditations métaphysiques. Oeuvres de Descartes. Paris: Vrin, 1996b. V. IX. . Las pasiones del alma. Madrid: Técnos, 1997.

DIACONU, M. Phänomenologie der Sinne. Stuttgart: Reclam, 2013.

FINK, E. Welt und Weltlichkeit. Würzburg: Königshausen \& Neumann, 1990.

GOLDSTEIN, B. Sensación y percepción. México D. F.: Thomson, 2005.

HEIDEGGER, M. Nietzsches Lehre vom Willen zur Macht als Erkenntnis. Frankfurt a. M.: Vitorio Klostermann, 1989. . Sein und Zeit. Tübingen: Max Niemeyer, 2001.

HENNING, H. Der Geruch. Leipzig: J.A. Barth, 1916.

HERING, E. Zur Lehre vom Lichtsinne. Viena: C. Gerold's Sohn, 1878.

HOFMANN, H. Untersuchungen über den Empfindungsbegriff. Archiv für die gesamte Psychologie. Leipzig, v. 26, p. 1-136, 1913.

HUSSERL, E. Ideen zu einer reinen Phänomenologie und philosophischen Philosophie. La Haya: Martinus Nijhoff, 1952. V. II.

. Ding und Raum: vorlesungen 1907. La Haya: Martinus Nijhoff, 1973.

. Logische Untersuchungen. Zweiter Band. I. Teil. Gesammelte Schriften 3.

Hamburg: Meiner, 1992a.

. Logische Untersuchungen. Zweiter Band. II. Teil. Gesammelte Schriften 4. Hamburg: Meiner, 1992b.

KATZ, D. Der Aufbau der Tastwelt. Leipzig: Johann Ambrosius Barth, 1925.

MARBACH, E. Das Problem des Ich in der Phänomenologie Husserls. Wien; New York: Springer, 1974.

MERLEAU-PONTY, M. Le primat de la perception et ses conséquences philosophiques. Paris: Gallimard, 1996. 
Phénoménologie de la perception. Paris: Gallimard, 2005.

MESSER, A. Empfindung und Denken. Leipzig: Quelle \& Meyer, 1908.

MUlligAN, K. Perception. In: SMITH, B. (Ed.). The Cambridge Companion to Husserl. Cambridge: Cambridge University Press, 1995. p. 168-322.

ORTEGA Y GASSET, J. Sobre el concepto de sensación. In: pensamiento. Madrid: Revista de Occidente, 1959. p. 73-98. Apuntes sobre el

PEUCKER, H. Von der Psychologie zur Phänomenologie: Husserls Weg in die Phänomenologie der 'Logischen Untersuchungen'. Hamburg: Meiner, 2002.

PLESSNER, H. Bei Husserl in Göttingen. In: Gesammelte Schriften. Ed. Günter Dux, Frankfurt a. M.: Suhrkamp, 1985. p. 344-354.V. IX.

SCHAPP, W. Erinnerungen an Edmund Husserl. Wiesbaden: B. Heymann, 1976. . In Geschichten verstrickt: zum Sein von Mensch und Ding. Frankfurt am Main: Vittorio Klostermann, 1985. . Beiträge zur Phänomenologie der Wahrnehmung. Frankfurt am Main: Vittorio Klostermann, 2013.

STRAUS, E. Vom Sinn der Sinne: ein Beitrag zur Grundlegung der Psychologie. Berlin; Heidelberg; New York: Springer, 1978.

WIESING, L. Das Mich der Wahrnehmung: eine Autopsie. Frankfurt a. M.: Suhrkamp, 2009.

Recebido: 24/03/2016

Aceito: 14/03/2017 
JOHNSON, F. 